\title{
On the Components of the Push-out Space with Certain Indices
}

\author{
YUSUF KaYA
}

ABSTRACT - Given an immersion of a connected, $m$-dimensional manifold $M$ without boundary into the Euclidean $(m+k)$-dimensional space, the idea of the push out space of the immersion under the assumption that immersion has flat normal bundle is introduced in [3]. It is known that the push-out space has finitely many path-connected components and each path-connected component can be assigned an integer called the index of the component. In this study, when $M$ is compact, we give some new results on the push-out space. Especially it is proved that if the push-out space has a component with index 1 , then the Euler number of $M$ is 0 and if the immersion has a co-dimension 2, then the number of path-connected components of the push-out space with index $(m-1)$ is at most 2 .

\section{Introduction}

Throughout we assume $M$ (or $M^{m}$ ) is an $m$-dimensional connected smooth $\left(\mathcal{C}^{\infty}\right)$ manifold without boundary. The tangent space of $M$ at a point $p$ will be denoted by $T_{p} M$.

$f: M^{m} \rightarrow \mathbb{R}^{m+k}$ will be assumed a smooth immersion or embedding into Euclidean $m+k$ space, i.e. $f$ has co-dimension $k$. In this case

$$
d f_{p}: T_{p} M^{m} \rightarrow T_{f(p)}\left(\mathbb{R}^{m+k}\right)=\{f(p)\} \times \mathbb{R}^{m+k} \cong \mathbb{R}^{m+k}
$$

is an injection. We identify $T_{p} M$ with $\operatorname{Im} d f_{p}, \forall p \in M$. In this way, we can assume that $f$ is an isometric immersion. There is a standard inner product $\langle$,$\rangle on \mathbb{R}^{m+k}$. So we can define the normal space at $p$ as the normal complement of $\operatorname{Im} d f_{p}$. Let $v_{p}(f)$ denote the $k$ - plane which is normal to $f(M)$ at

(*) Indirizzo dell'A.: Bülent Ecevit University, Faculty of Arts and Sciences, Department of Mathematics, 67100, Zonguldak/Turkey.

E-mail: yusufkaya69@gmail.com 
$f(p)$. The total space of the normal bundle is defined by

$$
N(f)=\left\{(p, v) \in M \times \mathbb{R}^{m+k}: f(p)+v \in v_{p}(f)\right\} .
$$

Note that $N(f)$ is an $(m+k)$-dimensional smooth manifold.

A normal field on $M$ for $f$ is a smooth map $\xi: M^{m} \rightarrow \mathbb{R}^{m+k}$ where $f(p)+\xi(p) \in v_{p}(f)$ for all $p \in M$.

With this notation, the endpoint map $E: N(f) \rightarrow \mathbb{R}^{m+k}$ is defined by $E(p, v)=f(p)+v$, and $E$ is known to be a smooth map.

\section{1 - Immersions of manifolds and focal points}

Definition 1. A point $x \in \mathbb{R}^{m+k}$ is a focal point of $f(M)$ with base $p$ if $E$ is singular at $(p, x-f(p))$, i.e. $(p, x-f(p))$ is a critical point of $E$. The focal point has multiplicity $\mu>0$ if rank (Jacobian $E)=m+k-\mu$.

The set of focal points of $f$ (or $f(M)$ ) with base $p$ will be denoted by $F_{p}(f)$.This is an algebraic variety, that is, it is a set of zeros of a polynomial with degree at most $m$ in $k$ variables in $v_{p}(f)$ and in general it can be quite complicated [8]. In this study, we will be considering the simplest case. We remark that by [6], $x \in F_{p}(f)$ iff $x \in v_{p}(f)$ and $x=f(p)+\frac{1}{\lambda} \xi(p)$ where $\xi(p)=\frac{x-f(p)}{\|x-f(p)\|}$ and $\lambda$ is an eigenvalue of the shape operator $A_{\xi(p)}: T_{p} M \longrightarrow T_{p} M$, i.e. $\lambda$ is a principal curvature of $f$ at $f(p)$ in the normal direction $\xi(p)$.

For $x \in \mathbb{R}^{m+k}$ the distance function for $f, L_{x}: M^{m} \rightarrow \mathbb{R}$ is defined by $L_{x}(p)=\|x-f(p)\|^{2}$. Using [6], the point $p \in M$ is a critical point of $L_{x}$ if and only if $x \in v_{p}(f)$ and further $p$ is a non-degenerate critical point of $L_{x}$ if and only if $x$ is not a focal point of $f$ with base $p$. So,

$$
F_{p}(f)=\left\{x \in \mathbb{R}^{m+k}: p \text { is a degenerate critical point of } L_{x}\right\} .
$$

We use this characterisation of $F_{p}(f)$ to calculate focal points with base $p$. Further, using [6] again, the index of $L_{x}$ at a non-degenerate critical point $p \in M$ is equal to the number of focal points of $f$ with base $p$ which lie on the line segment from $f(p)$ to $x$, each focal point being counted with its multiplicity.

\section{2 - Parallel immersions to a given immersion}

Definition 2. Let $f: M^{m} \rightarrow \mathbb{R}^{m+k}$ be an immersion and $\left\{\eta_{1}, \eta_{2}, \ldots, \eta_{k}\right\}$ be an orthonormal set of normal fields for $f$ in a neigh- 
bourhood of some point $p \in M$. A normal field $\xi$ for $f$ is said to be a parallel normal field, if $\left\langle\frac{\partial \xi}{\partial p_{i}}, \eta_{j}\right\rangle=0$ for all $p \in M$, where $i=1, \ldots, m, j=1, \ldots, k$ and $p_{1}, \ldots, p_{m}$ is a coordinate system in $a$ neighbourhood of $p \in M$.

Since we assume $M$ is connected, note that a parallel normal field on $M$ has constant length.

Let $f: M^{m} \rightarrow \mathbb{R}^{m+k}$ be an immersion and assume $\xi: M^{m} \rightarrow \mathbb{R}^{m+k}$ is a parallel normal field for $f$. The map $f_{\xi}: M^{m} \rightarrow \mathbb{R}^{m+k}$ is defined by

$$
f_{\xi}(p)=f(p)+\xi(p) .
$$

If $f_{\xi}$ is an immersion, it is called a parallel immersion to $f$ and $\xi$ is said to be immersive. We remark that, for all $p \in M$, the normal planes of $f$ and $f_{\xi}$ at each $p \in M$ are the same.

If $f_{\xi}$ is an immersion, then the index of $f_{\xi}$, ind $f_{\xi}$, is defined to be the total multiplicity of the focal points of $f$ with base $p$ on the line segment between $f(p)$ and $f_{\xi}(p)$, this index is shown to be constant over $M$ by the following well-known fact. We call this number as the index of the immersive parallel normal field $\xi$ as well.

LEMMA 1. Let $f: M^{m} \rightarrow \mathbb{R}^{m+k}$ be an immersion and let $\xi: M^{m} \rightarrow \mathbb{R}^{m+k}$ be a parallel normal field for $f$. Then the following are satisfied.

(i) $f_{\xi}$ is an immersion if and only if for all $p \in M, f_{\xi}(p)$ is not a focal point of $f$ with base $p$

(ii) $x \in \mathbb{R}^{m+k}$ is a focal point of $f_{\xi}$ with base $p$ if and only if $x$ is a focal point of $f$ with base $p$. So, $F_{p}\left(f_{\xi}\right)=F_{p}(f)$ for all $p \in M$.

\section{3 - The push-out space of immersions with flat normal bundle}

Let $M$ be a connected, $m$-dimensional manifold and $f: M^{m} \rightarrow \mathbb{R}^{m+k}$ be an immersion. If for all $p \in M$, there exists a neighbourhood $U \subset M$ of $p$ and a parallel normal frame field for $f$ on $U$, then it is said that $f$ has locally flat normal bundle. The normal bundle $N(f)$ is flat (or globally flat) if there exists a global parallel normal frame on $M$.

If the immersion $f$ has locally flat normal bundle, then at each base 
point $p \in M$, the focal set on $v_{p}(f)$ is a union of at most $m$ hyperplanes (which is the simplest set that can occur as focal set, if non empty) where each plane is counted with its proper multiplicity [8, pp. 69-70]. A generalisation and the converse of this result can be derived from [4].

First, we assume that the normal bundle of $f$ is globally flat. So there exists an orthonormal set of parallel normal fields $\xi_{1}, \ldots, \xi_{k}: M^{m} \rightarrow \mathbb{R}^{m+k}$ for $f$. For each $p \in M$, a map $\varphi_{p}: v_{p}(f) \rightarrow \mathbb{R}^{k}$ can be defined by $\varphi_{p}\left(f(p)+\sum_{i=1}^{k} a_{i} \xi_{i}(p)\right)=\left(a_{1}, \ldots, a_{k}\right)$. For each $p \in M$, we denote $\Omega_{p}=$ $\mathbb{R}^{k} \backslash \varphi_{p}\left(F_{p}(f)\right)$. Then, the push-out space of the immersion $f$ is defined by

$$
\Omega(f)=\bigcap_{p \in M} \Omega_{p} .
$$

This set is essentially defined and many properties of it are studied in [3]. For example, $\Omega(f)$ has finitely many path-connected components with each component convex and each component can be assigned an integer called as index. Further, if $M$ is compact, each component is open. The definition of $\Omega(f)$ depends on the choice of $\xi_{1}, \ldots, \xi_{k}$, but, it is shown in [3] that different choices produces an isometric set. We are going to study some properties of $\Omega(f)$ which are related to number of path-connected components of $\Omega(f)$ with certain indices and some relations with the Euler characteristic of $M$ (when $M$ is compact).

As pointed out in [3] we can next consider an immersion $f$ of $m$-dimensional manifold $M$ which has locally flat normal bundle but the normal holonomy group is nontrivial. In this case we can take the simply connected covering space $\tilde{M}$ of $M$ with covering map $\pi: \tilde{M}^{m} \rightarrow M^{m}$ and work with the immersion $\tilde{f}=f \circ \pi: \tilde{M}^{m} \rightarrow \mathbb{R}^{m+k}$ which has globally flat normal bundle with trivial normal holonomy. We know that $f$ and $\tilde{f}$ have the same focal set:

Proposition 1. With the notation above, $F_{p}(f)=F_{\tilde{p}}(\tilde{f})$ for all $p \in M$ and $\tilde{p} \in \tilde{M}$ with $\pi(\tilde{p})=p$, where $\pi: \tilde{M} \longrightarrow M$ is the covering map.

Proof. Let $x \in \mathbb{R}^{m+k}$ and define $\tilde{L}_{x}: \tilde{M}^{m} \rightarrow \mathbb{R}$ (distance function for the immersion $\tilde{f}$ ) by

$$
\tilde{L}_{x}(\tilde{p})=\|x-\tilde{f}(\tilde{p})\|^{2}=L_{x} \circ \pi(\tilde{p}),
$$

where $L_{x}: M^{m} \rightarrow \mathbb{R}$ is the usual distance function for $f$. Since $\pi$ is an im- 
mersion, $\tilde{p}$ is a degenerate critical point of $\tilde{L}_{x}$ if and only if $\pi(\tilde{p})$ is a degenerate critical point of $L_{x}$. Therefore $F_{p}(f)=F_{\tilde{p}}(\tilde{f})$ for all $\tilde{p} \in \tilde{M}$ and $p \in M$ with $\pi(\tilde{p})=p$.

So this result allows $\Omega(f)$ to be defined by $\Omega(f)=\Omega(f \circ \pi)=\Omega(\tilde{f})$. This is useful especially when we have an immersion of a nonorientable manifold with locally flat normal bundle where obviously the normal holonomy group is nontrivial. Consequently, by replacing $f$ with $\tilde{f}$ if necessary, we may assume that $f$ has globally flat normal bundle with trivial normal holonomy group. Remark that $\tilde{M}$ may fail to be compact again even $M$ is compact, but we can use critical point theory of distance function thorough the immersion of $M$ to deduce some results on $\Omega(\tilde{f})$.

Let $a=\left(a_{1}, a_{2}, \ldots, a_{k}\right) \in \Omega(f)$. As in [3], define $\xi(a): M^{m} \rightarrow \mathbb{R}^{m+k}$ by $\xi(a)(p)=\sum_{i=1}^{k} a_{i} \xi_{i}(p)$, where $\xi_{1}, \xi_{2}, \ldots, \xi_{k}$ are unit parallel normal fields on $M$ forming a basis for the normal $k$-plane at $f(p)$ for all $p \in M$. Then it is easy to check that $\xi(a)$ is an immersive parallel normal field for $f$ on $M$. With this notation $\Omega(f)$ can be defined as

$\Omega(f)=\left\{a \in \mathbb{R}^{k}: f(p)+\xi(a)(p)\right.$ is not a focal point of $f$ with base $\left.p, \forall p \in M\right\}$.

Definition 3. Let $a \in \Omega(f)$. The index of $a$, ind $a$, is defined to be the index of the immersion $f_{\xi(a)}$.

We know by [3] that if $A$ is a path-connected component of $\Omega(f)$ and if $a, b \in A$, then ind $a=$ ind $b$. Then the index of $A$ is defined to be ind $a$ for some $a \in A$ which is constant over $A$. So each path-connected component of $\Omega(f)$ can be assigned a number, called its index. We will denote the union of the path-connected components of $\Omega(f)$ with index $\mu$ by $\Omega^{\mu}$. So $\Omega(f)=\Omega^{0} \cup \Omega^{1} \cup \cdots \cup \Omega^{m}$. Note that $\Omega^{0}$ is always non empty and the others may be empty or not.

\section{Path-connected components and their respective indices.}

In this section, firstly, we give an example to illustrate the $\Omega(f)$ for a given embedding $f$ with flat normal bundle and then we prove some general results on $\Omega(f)$. 
ExAmple 1. Let $\tilde{f}: \mathbb{R} \times \mathbb{R} \rightarrow \mathbb{S}^{3} \subset \mathbb{R}^{2+2}$ be given by

$$
\tilde{f}(\theta, \phi)=\frac{1}{\sqrt{2}}(\cos \theta, \sin \theta, \cos \phi, \sin \phi),
$$

then $\tilde{f}$ induces an embedding $f$ of $\mathbb{S}^{1} \times \mathbb{S}^{1}$ into $\mathbb{S}^{3} \subset \mathbb{R}^{2+2}$ by taking $\theta$ $\bmod 2 \pi, \phi \bmod 2 \pi$ and also $\Omega(f)=\Omega(\tilde{f})$. Now,

$$
\begin{aligned}
& \xi_{1}(\theta, \phi)=\tilde{f}(\theta, \phi)=\frac{1}{\sqrt{2}}(\cos \theta, \sin \theta, \cos \phi, \sin \phi), \\
& \xi_{2}(\theta, \phi)=\frac{1}{\sqrt{2}}(-\cos \theta,-\sin \theta, \cos \phi, \sin \phi)
\end{aligned}
$$

are unit parallel normal fields to $\tilde{f}$ and form a basis for the normal planes for all $(\theta, \phi) \in \mathbb{R} \times \mathbb{R}$. Put $\xi(\theta, \phi)=t \xi_{1}(\theta, \phi)+s \xi_{2}(\theta, \phi)$, for some $t, s \in \mathbb{R}$, then

$$
\begin{aligned}
\tilde{f}_{\xi}(\theta, \phi) & =\tilde{f}(\theta, \phi)+t \xi_{1}(\theta, \phi)+s \xi_{2}(\theta, \phi) . \\
& =\frac{1}{\sqrt{2}}((1+t-s) \cos \theta,(1+t-s) \sin \theta,(1+t+s) \cos \phi,(1+t+s) \sin \phi) .
\end{aligned}
$$

Using the distance function $L_{x}(\theta, \phi)=\|x-\tilde{f}(\theta, \phi)\|^{2}$ for $x=\left(x_{1}, x_{2}, x_{3}, x_{4}\right) \in$ $\mathbb{R}^{4}$, we get

$$
\begin{array}{cc}
\frac{\partial L_{x}}{\partial \theta}=\frac{2}{\sqrt{2}}\left(x_{1} \sin \theta-x_{2} \cos \theta\right), & \frac{\partial L_{x}}{\partial \phi}=\frac{2}{\sqrt{2}}\left(x_{3} \sin \phi-x_{4} \cos \phi\right), \\
\frac{\partial^{2} L_{x}}{\partial \theta^{2}}=\frac{2}{\sqrt{2}}\left(x_{1} \cos \theta+x_{2} \sin \theta\right), & \frac{\partial^{2} L_{x}}{\partial \phi^{2}}=\frac{2}{\sqrt{2}}\left(x_{3} \cos \theta+x_{4} \sin \theta\right), \\
\frac{\partial^{2} L_{x}}{\partial \phi \partial \theta}=\frac{\partial^{2} L_{x}}{\partial \theta \partial \phi}=0 .
\end{array}
$$

Then

$$
\operatorname{Hess}\left(L_{x}\right)=H=\left[\begin{array}{cc}
\frac{\partial^{2} L_{x}}{\partial \theta^{2}} & 0 \\
0 & \frac{\partial^{2} L_{x}}{\partial \phi^{2}}
\end{array}\right]
$$

So $\tilde{f}_{\xi}(\theta, \phi)$ is a focal point of $\tilde{f}$ at $(\theta, \phi) \Longleftrightarrow(\theta, \phi)$ is a degenerate critical point of $L_{x}$. From the equations $\frac{\partial L_{x}}{\partial \theta}=0=\frac{\partial L_{x}}{\partial \phi}$, we obtain, for each $(\theta, \phi) \in \mathbb{R}^{2}$, $x=\tilde{f}_{\xi}(\theta, \phi)$ for some $t, s \in \mathbb{R}$. By replacing $x$ by $\tilde{f}_{\xi}(\theta, \phi)$ and using $\operatorname{det} H=0$ 
we get

$$
\begin{aligned}
\operatorname{det}\left[\begin{array}{cc}
\frac{2}{\sqrt{2}}(1+t-s) & 0 \\
0 & \frac{2}{\sqrt{2}}(1+t+s)
\end{array}\right]=0 & \Longleftrightarrow(1+t+s)(1+t-s)=0 \\
& \Longleftrightarrow(1+t)^{2}-s^{2}=0 \\
& \Longleftrightarrow s= \pm(1+t) .
\end{aligned}
$$

Therefore the focal set of $\tilde{f}$ with base $(\theta, \phi) \in \mathbb{R} \times \mathbb{R}$ is a pair of lines perpendicular to one another which is the same for all base points $(\theta, \phi)$. Consequently

$$
\Omega(\tilde{f})=\Omega_{(\theta, \phi)}(\tilde{f})=\Omega_{\left(\theta^{\prime}, \phi^{\prime}\right)}(\tilde{f}), \quad \forall(\theta, \phi),\left(\theta^{\prime}, \phi^{\prime}\right) \in \mathbb{R} \times \mathbb{R} .
$$

Then, $\Omega(\tilde{f})$ has four path-connected components since each $\Omega_{(\theta, \phi)}(\tilde{f})$ has four path-connected components; one of index 0 , two of index 1 , and one of index 2. Hence the same is true for $\Omega(f)$, as $\Omega(f)=\Omega(\tilde{f})$, see the Figure 1. Then $\Omega(f)=\Omega^{0} \cup \Omega^{1} \cup \Omega^{2}$ and in the Figure 1, we put $\Omega^{1}=A \cup B$.

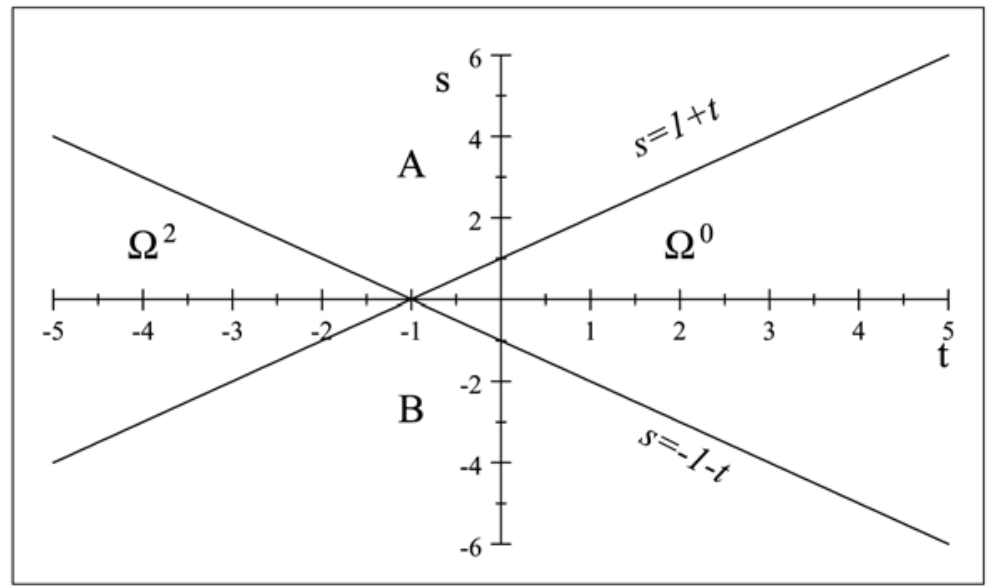

Figure 1

We know by [3], $\Omega(f)$ can have at most one component with index $m$ and if there is such a component, it is unbounded. Note that, we have examples of immersions such that $\Omega^{0}$ is bounded. 
THEOREM 1. Let $f: M^{m} \rightarrow \mathbb{R}^{m+k}$ be an immersion of a compact, $m$ dimensional manifold such that $f$ has flat normal bundle. If $\Omega(f)$ has a component with index $m$, then $\Omega^{0}$ is unbounded.

Proof. Let $a \in \Omega^{m}$ and take the immersive parallel normal field $\xi(a)$. Then $\varphi_{p}^{-1}(a)=f_{\xi(a)}(p), \forall p \in M$ and index $f_{\xi(a)}=m$. So for all $p \in M$, the total multiplicity of focal points with base $p$ on the line segment from $f(p)$ to $f_{\xi(a)}(p)$ is $m$. Therefore there are no focal points on the rays

$$
\begin{aligned}
& R_{p}=\left\{f(p)+t \xi(a)(p) \in v_{p}(f): t \geq 1\right\} \\
& Q_{p}=\left\{f(p)+t \xi(a)(p) \in v_{p}(f): t \leq 0\right\} .
\end{aligned}
$$

Also $\forall p \in M, \varphi_{p}\left(Q_{p}\right)=\{t a: t \leq 0\} \subset \Omega_{p}$ and so

$$
\{t a: t \leq 0\} \subset \bigcap\left\{\Omega_{p}: p \in M\right\}=\Omega(f) .
$$

Hence $\{t a: t \leq 0\} \subset \Omega^{0}$ and $\Omega^{0}$ is unbounded.

Proposition 2. Let $f: M^{m} \rightarrow \mathbb{R}^{m+k}$ be an immersion with flat normal bundle and assume $\xi, \eta: M^{m} \rightarrow \mathbb{R}^{m+k}$ are immersive parallel normal fields for $f$ with indices $\lambda$ and $\mu$ respectively. Then, the number offocal points with base $p$ on the line segment from $f_{\xi}(p)$ to $f_{\eta}(p)$ is constant for all $p \in M$ and it is $\lambda+\mu-2 l$ for some $l \in \mathbb{N}$ where $\max \{0, \lambda+\mu-m\} \leq l \leq \min \{\lambda, \mu\}$.

Proof. As in Lemma 4.4 of [3], since $f_{\xi}+(\eta-\xi)=f_{\eta}$, so $\eta-\xi$ is an immersive parallel normal field for the immersion $f_{\xi}$ and we need to find its index for $f_{\xi}$ which is constant. Here we try to formulate this constant.

Let $p \in M$ and put $x=f_{\xi}(p)$ and $y=f_{\eta}(p)$. If $f(p), x, y$ are collinear, then the number of focal points with base $p$ on the line segment from $f_{\xi}(p)$ to $f_{\eta}(p)$ is $\lambda+\mu$ or $|\lambda-\mu|$ with respect to positioning of $f(p)$ and we can take $l=0$ or $l=\lambda$ or $l=\mu$. Otherwise, take the triangle on $v_{p}(f)$ with vertices $f(p), x, y$, and consider the 2 -plane say $Q(p)$ which contains this triangle. We know that $Q(p) \cap F_{p}(f)$ is a union of at most $m$ lines if it is non empty, since $F_{p}(f)$ is a union of at most $m$ hyperplanes on $v_{p}(f)$ [8].

If $u, v \in v_{p}(f)$, then the notation $\overline{u v}$ denotes the line segment from $u$ to $v$. We know the total multiplicity of focal points on $\overline{f(p) x}$ is $\lambda$ and on $\overline{f(p) y}$ is $\mu$. Now, let $l(p) \geq 0$ be an integer and assume that $l(p)$ lines(counting multiplicities) meet both of the edges $\overline{f(p) x}$ and $\overline{f(p) y}$. Clearly $0 \leq l(p) \leq \min \{\lambda, \mu\}$. Then the remaining $\lambda-l(p)$ lines intersecting $\overline{f(p) x}$ must intersect $\overline{x y}$. And similarly the remaining $\mu-l(p)$ lines intersecting $\overline{f(p) y}$ must intersect $\overline{x y}$. So we get the total multiplicity on $\overline{x y}$ is exactly 
$\lambda-l(p)+\mu-l(p)=\lambda+\mu-2 l(p)$. So we deduce the total multiplicity of focal points on the line segment from $f_{\xi}(p)$ to $f_{\eta}(p)$ is $\lambda+\mu-2 l(p)$. But the index of the parallel immersion $\left(f_{\xi}\right)_{(\eta-\xi)}$ to $f_{\xi}$ is a constant number, so $l(p)$ is constant for all $p \in M$.

Put $l=l(p)$. Since there exist at most $m$ lines on $Q(p)$, then $\lambda+\mu-m \leq l$. Then $\max \{0, \lambda+\mu-m\} \leq l \leq \min \{\lambda, \mu\}$.

Corollary 1. If $\lambda=\mu=1$ in Proposition 2, then, for all $p \in M$, the number of focal points with base $p$ on the line segment from $f_{\xi}(p)$ to $f_{\eta}(p)$ is 2 (where $l=0)$.

THEOREM 2. Let $f: M^{m} \rightarrow \mathbb{R}^{m+k}$ be an immersion with flat normal bundle and assume $\xi: M^{m} \rightarrow \mathbb{R}^{m+k}$ is an immersive parallel normal field for $f$.

(i) There exists a $w \in \mathbb{R}^{k}$ such that $\Omega\left(f_{\xi}\right)=\Omega(f)-w$, where $\Omega(f)-w=\{a-w: a \in \Omega(f)\}$,

(ii) if $A$ is a path-connected component of $\Omega(f)$ with index $\mu$ and if the index of $f_{\xi}$ is $\lambda$, then there exists an $l \in \mathbb{N}$ such that $A-w$ is a pathconnected component of $\Omega\left(f_{\xi}\right)$ with index $\lambda+\mu-2 l$, where $\max \{0$, $\lambda+\mu-m\} \leq l \leq \min \{\lambda, \mu\}$.

Proof. (i) Since $f$ has flat normal bundle, there exists a set of orthonormal parallel normal fields $\left\{\xi_{1}, \xi_{2}, \cdots, \xi_{k}\right\}$ forming a basis of the normal space at each base point $p \in M$. We will use this basis to define $\Omega(f)$ and $\Omega\left(f_{\xi}\right)$. We can write

$$
\xi=w_{1} \xi_{1}+\cdots+w_{k} \xi_{k}
$$

for some constants $w_{1}, \ldots, w_{k} \in \mathbb{R}$ and put $w=\left(w_{1}, \ldots, w_{k}\right) \in \mathbb{R}^{k}$. Then we can easily see that $a \in \Omega(f) \Longleftrightarrow a-w \in \Omega\left(f_{\xi}\right)$. In fact, let $a=\left(a_{1}, \ldots, a_{k}\right) \in \Omega(f)$. We know $F_{p}(f)=F_{p}\left(f_{\xi}\right)$ for all $p \in M$ by Lemma 1 (ii). Then, for all $p \in M$

$$
\begin{aligned}
f(p)+a_{1} \xi_{1}+\cdots+a_{k} \xi_{k} & \notin F_{p}(f) \Longleftrightarrow f+\xi+a_{1} \xi_{1}+\cdots+a_{k} \xi_{k}-\xi \\
& =f_{\xi}+\left(a_{1}-w_{1}\right) \xi_{1}+\cdots+\left(a_{k}-w_{k}\right) \xi_{k} \notin F_{p}\left(f_{\xi}\right) .
\end{aligned}
$$

So $a-w \in \Omega\left(f_{\xi}\right)$ and therefore $\Omega\left(f_{\xi}\right)=\Omega(f)-w$.

(ii) Let $a \in A$, then clearly $a-w \in \Omega\left(f_{\xi}\right)$ by Theorem 2 (i), hence $A-w$ is a path-connected component of $\Omega\left(f_{\xi}\right)$. Since $A$ is a path-connected component of $\Omega(f)$ with index $\mu$, there exists an immersive parallel normal field $\eta$ for $f$ with index $\mu$ and $\varphi_{p}(f(p)+\eta(p))=a$ for all $p \in M$. As in 
Proposition $2, f_{\xi}+(\eta-\xi)=f_{\eta}$, so $\eta-\xi$ is an immersive parallel normal field for $f_{\xi}$ and its index for $f_{\xi}$ is $\lambda+\mu-2 l$ for some $l \in \mathbb{N}$ where $\max \{0, \lambda+\mu-m\} \leq l \leq \min \{\lambda, \mu\}$.

The following result concerns the positioning of the path-connected components of $\Omega(f)$ in $\mathbb{R}^{k}$.

THEOREM 3. Let $f: M^{m} \rightarrow \mathbb{R}^{m+k}$ be an immersion with flat normal bundle. Let $A, B$ be path-connected components of $\Omega(f)$ with index $\lambda$ and $\mu$ respectively. If $\lambda+\mu>m$, then there exists a hyperplane in $\mathbb{R}^{k}$ such that $A$ and $B$ lie on one side of the hyperplane and $\Omega^{0}$ lies on the opposite side of the hyperplane.

Proof. Let $A, B$ be path-connected components of $\Omega(f)$ with index $\lambda$ and $\mu$ respectively and $a \in A, b \in B$. Then there exist immersive parallel normal fields $\xi, \eta$ for $f$ such that index $f_{\xi}=\lambda$, index $f_{\eta}=\mu$ and also for all $p \in M, \varphi_{p}^{-1}(a)=f_{\xi}(p)$ and $\varphi_{p}^{-1}(b)=f_{\eta}(p)$. Now consider the normal plane $v_{p}(f)$ for a fixed $p \in M$ and the focal hyperplanes $\Pi_{1}, \ldots, \Pi_{s}$ on $v_{p}(f)$ with their respective multiplicity $w_{i}$ where $1 \leq i \leq s, s \leq m$ and $w_{1}+\cdots+w_{s} \leq m$

Since $f_{\xi}$ has index $\lambda$, the line segment joining $f(p)$ to $f_{\xi}(p)$ must cross $\Pi_{\alpha_{1}}, \ldots, \Pi_{\alpha_{l}}$ where $l \leq \lambda, w_{\alpha_{1}}+\cdots+w_{\alpha_{l}}=\lambda$ and similarly the line segment joining $f(p)$ to $f_{\eta}(p)$ must cross $\Pi_{\beta_{1}}, \ldots, \Pi_{\beta_{d}}$ where $d \leq \mu$, $w_{\beta_{1}}+\cdots+w_{\beta_{d}}=\mu$.

Here, $\Pi_{\alpha_{1}}, \ldots, \Pi_{\alpha_{l}}, \Pi_{\beta_{1}}, \ldots, \Pi_{\beta_{d}}$ are not all distinct since $\lambda+\mu>m$. So let $\Pi \in\left\{\Pi_{\alpha_{1}}, \ldots, \Pi_{\alpha_{l}}\right\} \cap\left\{\Pi_{\beta_{1}}, \ldots, \Pi_{\beta_{d}}\right\}$. Then we claim that $A, B$ stay on one side of the hyperplane $\varphi_{p}(\Pi)=\Lambda$ in $\mathbb{R}^{k}$. Set $\varphi_{p}\left(\Pi_{i}\right)=\Lambda_{i}, 1 \leq i \leq s$. Since each $\Lambda_{i}$ divides $\mathbb{R}^{k}$ into two open connected regions, we identify them by writing $\Lambda_{i}^{-}$for the region including the origin and $\Lambda_{i}^{+}$for the other part.

Then, $\Omega^{0} \subset \Lambda_{i}^{-} \quad$ for all $1 \leq i \leq s, \quad A \subset \Lambda_{\alpha_{1}}^{+} \cap \cdots \cap \Lambda_{\alpha_{l}}^{+}$and $B \subset$ $\Lambda_{\beta_{1}}^{+} \cap \cdots \cap \Lambda_{\beta_{d}}^{+}$. Therefore $A$ and $B$ stay in $\Lambda^{+}$, and hence $\Lambda$ is the hyperplane we are seeking.

\section{Number of path-connected components of $\boldsymbol{\Omega}(f)$ with certain indices}

It is interesting to know the number of path-connected components of $\Omega(f)$ with their respective indices for an immersion $f$ of $M$ as it includes some information on the geometry and the topology of the $m$-dimensional compact manifold $M$. Here, we prove that if we have a path-connected 
component of $\Omega(f)$ with index 1, then the Euler characteristic of $M$ is 0 . Secondly, we prove that the number of path-connected components of $\Omega(f)$ with index $(m-1)$ is at most 2 for a co-dimension 2 immersion.

THEOREM 4. Let $f: M^{m} \longrightarrow \mathbb{R}^{m+k}$ be an immersion of compact manifold with flat normal bundle and let $\chi(M) \neq 0$ where $m$ is an even number. Then $\Omega^{1}=\emptyset$.

PRoof. If $\Omega^{1} \neq \emptyset$, then there exists a unit parallel normal field $\xi$ for $f$ such that $f_{s \xi}=f+s \xi$ is an immersion with index 1 for some $s>0$. So $\forall p \in M$, there exists only one focal point $c(p)$ of multiplicity 1 on the line segment from $f(p)$ to $f_{s \xi}(p)$ such that $c: M^{m} \longrightarrow \mathbb{R}^{m+k}, p \rightarrow c(p)$ is continuous. Define $\lambda: M^{m} \longrightarrow \mathbb{R}$ by

$$
\lambda(p)=\frac{1}{\|f(p)-c(p)\|} .
$$

Then $\lambda$ is continuous as it is the principal curvature function of $f$ in the unit normal direction $\xi$. Also $\lambda$ is smooth since it is of constant multiplicity 1 on $M$ [7]. So the principal direction corresponding to the principal curvature $\lambda(p)$ defines a nonzero smooth tangent vector field on $M$ which has no zeros. So considering that $M$ is compact, $\chi(M)=0$ by the Poincaré-Hopf Theorem in [5]. But this contradicts $\chi(M) \neq 0$. Therefore $\Omega^{1}=\emptyset$.

A generalisation of this theorem to any odd indexed component is proved in [1] by a different method. Present method here may not be generalized, because respective vector field can fail to be smooth.

DEFINITION 4. Let $f: M^{m} \longrightarrow \mathbb{R}^{m+k}$ be an immersion with flat normal bundle, then $d(f)$ is defined to be the total number of the path-connected components of $\Omega(f)$.

It was proved in [3] that $d(f) \leq \alpha(m, k)$ where $\alpha(m, k)$ is the number of path-connected regions in the complement of $m$ hyperplanes in general position in $\mathbb{R}^{k}$ as

$$
\alpha(m, k)=\left\{\begin{array}{ll}
2^{m} & \text { if } m \leq k \\
\sum_{i=0}^{k}\left(\begin{array}{c}
m \\
i
\end{array}\right) & \text { if } m>k
\end{array} .\right.
$$


COROLlary 2. Let $f: M^{2} \longrightarrow \mathbb{R}^{2+k}$ be an immersion with flat (or locally flat) normal bundle of a compact surface for some $k \geq 1$ and let $\chi(M) \neq 0$. Then $\Omega^{1}=\emptyset$ and so $d(f) \leq 2$.

Proof. By Theorem $4, \Omega^{1}=\emptyset$ and also $\Omega^{0}, \Omega^{2}$ are connected [3], hence $d(f) \leq 2$. Of course $\Omega^{2}$ can occur, definitely when $f$ is spherical, [2].

EXAmPLE 2. For the homology groups of real projective space $\mathbb{R} P^{m}$, we know that $H_{i}\left(\mathbb{R} P^{m}, Z_{2}\right)=Z_{2}$ for all $i=1,2, \ldots, m$. Then

$$
\chi\left(R P^{m}\right)=\left\{\begin{array}{ll}
0, & \text { if } m \text { is odd } \\
1, & \text { if } m \text { is even }
\end{array} .\right.
$$

So, by Theorem 4 , if $f: \mathbb{R} P^{m} \longrightarrow \mathbb{R}^{m+k}$ is any immersion with locally flat normal bundle, we have $\Omega^{1}=\emptyset$ for $m$ is even.

Let $f: M^{2} \longrightarrow \mathbb{R}^{2+k}$ be an immersion of a 2 -dimensional manifold $M$ with flat normal bundle. Then for any point $p \in M, F_{p}(f)$ is a union of at most 2 hyperplanes in $v_{p}(f)$. So $F_{p}(f)$ can divide $v_{p}(f)$ into at most 4 pathconnected regions, and the number of path-connected components of $\Omega(f)$ with index 1 can be at most 2 for any $k \geq 2$.

In the following theorems we generalize this and prove a result concerning the number of path-connected components of $\Omega(f)$ with index $(m-1)$ where $m \geq 3$.

THEOREM 5. Let $m \geq 2$ and $f: M^{m} \longrightarrow \mathbb{R}^{m+k}$ be an immersion with flat normal bundle. Assume $A, B$ are two different path-connected components of $\Omega(f)$ both with index $(m-1)$ and $a \in A, b \in B$. Then for each $p \in M$, all the focal hyperplanes in $v_{p}(f)$ meet the triangle $\triangle$ with vertices $f(p), \varphi_{p}^{-1}(a), \varphi_{p}^{-1}(b)$, and moreover the total number of focal points on the line segment from $\varphi_{p}^{-1}(a)$ to $\varphi_{p}^{-1}(b)$ is exactly 2 .

Proof. Let $a \in A, b \in B$. Then there are corresponding parallel normal fields $\xi=\xi(a)$ and $\eta=\xi(b)$ say, such that index $f_{\xi}=$ index $f_{\eta}=m-1$. By Proposition 2, for all $p \in M$, we have total number of focal points between $f_{\xi}(p)=\varphi_{p}^{-1}(a)$ and $f_{\eta}(p)=\varphi_{p}^{-1}(b)$ is $2(m-1)-2 l$ for some $l \in \mathbb{N}$ where $m-2 \leq l \leq m-1$. Since $a$ and $b$ are in different components, there must be at least one focal point between $f_{\xi}(p)$ and $f_{\eta}(p)$ for all $p \in M$. So $l=m-2$. 
Let $Q \subset v_{p}(f)$ be the plane including the triangle $\triangle$ with vertices $f(p)$, $f_{\xi}(p), f_{\eta}(p)$. Since $l=m-2$, we have proved that the total multiplicity of focal points on $\overline{f_{\xi}(p) f_{\eta}(p)}$ is exactly 2 for all $p \in M$ and hence there are exactly $m$ focal lines meeting with the triangle $\triangle$ as required. Since there are $m$ lines in $Q$, this implies that all focal hyperplanes on $v_{p}(f)$ meet with the triangle $\triangle$, for all $p \in M$.

THEOREM 6. Let $f: M^{m} \longrightarrow \mathbb{R}^{m+2}$ be an immersion of a compact manifold such that $f$ has flat normal bundle, where $m \geq 3$. Then the number of path-connected components of $\Omega(f)$ with index $(m-1)$ is at most 2.

Proof. Assume there exist at least three path-connected components of $\Omega(f)$ with index ( $m-1)$, say $A, B, C$. Take $a \in A, b \in B, c \in C$. Let $p \in M$ be an arbitrary point and consider the points $x=\varphi_{p}^{-1}(a), y=\varphi_{p}^{-1}(b)$, $z=\varphi_{p}^{-1}(c)$ on $v_{p}(f)$. Clearly $x, y, z$ are nonfocal distinct points, since $a, b, c$ are in different components.

Since $a, b, c$ are in different components there is at least one focal point on each line segment $\overline{x y}, \overline{y z}, \overline{z x}$. So we can check that the points $x, y, f(p)$ cannot be collinear. Assume they lie on a line $\ell$ say. If $f(p)$ is on $\overline{x y}$ then the total multiplicity of focal points on $\ell$ is at least $(2 m-2)$ which is not possible for $m \geq 3$, since $2 m-2>m$. If $f(p)$ is not on $\overline{x y}$ we get the total multiplicity of focal points on $\overline{f(p) x}$ or $\overline{f(p) y}$ is at least $m$ depending on the positioning of $f(p)$ on $\ell$ with respect to the points $x, y$. This contradicts the hypothesis that this number is $(m-1)$. By a similar discussion we get the points $x, z, f(p)$ or $y, z, f(p)$ or $x, y, z, f(p)$ cannot be collinear.

By Theorem 5, all of the focal lines must meet the triangle with vertices $x, y, f(p)$ and further the total multiplicity of focal points on $\overline{x y}$ is exactly 2 . Similarly we get the same result considering the triangles with vertices $y, z, f(p)$ and $x, z, f(p)$.

We next show $x, y, z$ are not collinear. For if $x, y, z$ all lie on a line then by the above argument the total multiplicity of focal points on each line segment $\overline{x y}, \overline{y z}, \overline{z x}$ is exactly 2 . Without loss of generality we can assume $y$ is on $\overline{x z}$. Then we obtain the total multiplicity of focal points on $\overline{x z}$ is $2+2=4$ which is a contradiction.

Now consider the triangle with vertices $x, y, z$. There are 3 cases to be considered.

CASE 1. Assume $f(p)$ is in the region I bounded by the triangle with vertices $x, y, z$ as shown in Figure 2. By Theorem 5 there exists at least one focal line meeting with $\overline{x f(p)}$ and $\overline{z f(p)}$ considering the triangle with ver- 
tices $x, f(p), z$. Similarly there exists one focal line meeting with $\overline{x f(p)}$ and $\overline{y f(p)}$ considering the triangle with vertices $x, f(p), y$. And also there exists one focal line meeting with $\overline{y f(p)}$ and $\overline{z f(p)}$ considering the triangle with vertices $y, f(p), z$. These focal lines are necessarily all different and together bound $f(p)$. This implies that $f(p)$ is in a bounded region of the complement of the focal lines on $v_{p}(f)$.

CASE 2. Assume $f(p)$ is in the region II as shown in Figure 2. Consider the triangle with vertices $z, f(p), y$. By Theorem 5 , there must be a focal line meeting with $\overline{f(p) z}$ and $\overline{z y}$ and this line must necessarily meet $\overline{x f(p)}$ and $\overline{x y}$. Similarly by considering the triangle with vertices $x, f(p), z$, there must be a focal line meeting with $\overline{f(p) z}$ and $\overline{z x}$ and this line must necessarily meet $\overline{f(p) y}$ and $\overline{x y}$. Now we get at least 2 focal points on $\overline{x y}$. But again by Theorem 5 and considering the triangle with vertices $x, f(p), y$, it is exactly 2 . So there are no more focal lines meeting with $\overline{x y}$. So far we have one focal line meeting both $\overline{x f(p)}$ and $\overline{z f(p)}$. By Theorem 5 and considering the triangle with vertices $x, f(p), z$, we need $(m-3)$ more focal lines meeting with $\overline{x f(p)}$ and $\overline{z f(p)}$ which must necessarily meet with $\overline{y f(p)}$. And one more focal line meeting both $\overline{x f(p)}$ and $\overline{x z}$ which must necessarily meet with $\overline{x y}$ or $\overline{z y}$. We know there are no more focal lines meeting with $\overline{x y}$. So the focal line meeting both $\overline{x f(p)}$ and $\overline{x z}$ must necessarily meet with $\overline{z y}$. This implies that for all $p \in M, z=f_{\xi(a)}(p)$ is bounded by focal lines on $v_{p}(f)$ where the immersive parallel normal field $\xi(a)$ is corresponding to $a$.

CASE 3. Now assume $f(p)$ is in the region III as shown in Figure 2. Then we know every focal line must meet the triangle with vertices $f(p), x, y$. But there must be a focal line meeting with $\overline{f(p) z}$ and $\overline{z x}$ simultaneously. So this line cannot meet the triangle with vertices $f(p), x, y$. This gives a contradiction by Theorem 5 . So we deduce that Case 3 cannot occur.

Since $p$ is an arbitrary point in $M$ and $\varphi_{p}{ }^{-1}$ is an isometry, then either Case 1 holds for all $p \in M$ or Case 2 holds for all $p \in M$ i.e. either $f(p)$ is bounded by focal lines on $v_{p}(f)$ or $f_{\xi(a)}(p)$ is bounded by focal lines on $v_{p}(f)$ for all $p \in M$.

Now, for some $w \in \mathbb{R}^{m+2}$, take the distance function $L_{w}$ for $f$. Since $M$ is compact, there is a critical point of $L_{w}$ with index $m$. So the total number of focal points with base $p$ on the line segment from $w$ to $f(p)$ is $m$ and so there is no focal point with base $p$ on the ray $\{f(p)+t(w-f(p)) \mid t \leq 0\} \subset v_{p}(f)$. This implies that for some $p \in M, f(p)$ is not bounded by focal hyperplanes on $v_{p}(f)$ and a similar statement is true for $f_{\xi(a)}(q)$ considering the immersion 
$f_{\xi(a)}$ for some $q \in M$. So there cannot be such path-connected components $A, B, C$ of $\Omega(f)$. Therefore $\Omega(f)$ can have at most two path-connected components with index $(m-1)$.

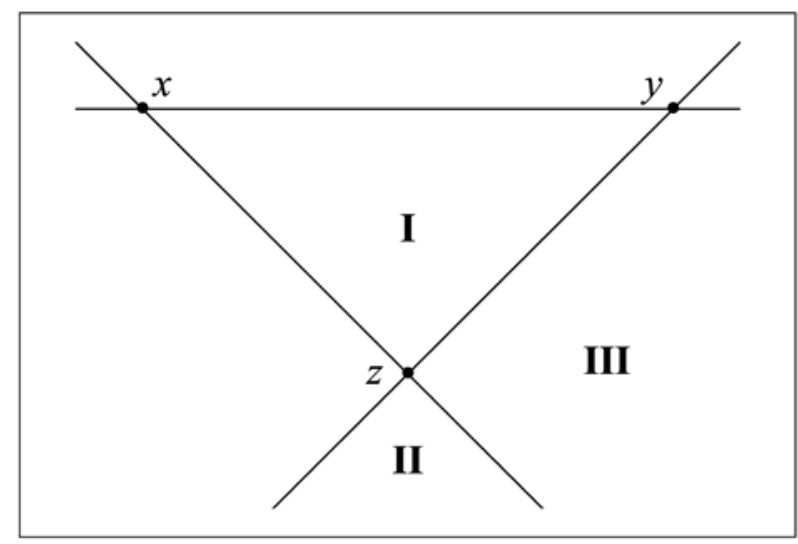

Figure 2

REMARK 1. Theorem 6 is not true for an immersion with co-dimension $k>2$. We can see that by taking product immersions. In Example 1, we have an embedding $f$ of $\mathbb{T}^{2}$ into $\mathbb{S}^{3} \subset \mathbb{R}^{4}$ such that $\Omega(f)$ has two pathconnected components with index 1 . Now take

$$
f \times f:{ }^{2} \times T^{2} \longrightarrow \mathbb{R}^{4+4}
$$

by $(f \times f)(p, q)=(f(p), f(q))$ where $p, q \in T^{2}$. Note that, by Theorem 4.2 of [3], $f \times f$ has flat normal bundle and $\Omega(f \times f)=\Omega(f) \times \Omega(f)$, since $f$ has flat normal bundle. Then, we can easily check that $\Omega(f \times f)$ has 4 pathconnected components with index 3 .

Consequently, for $m>2$ and $k>2$, it is a considerable question to ask what is the maximum number of path-connected components of $\Omega(f)$ with index $(m-1)$. This might be at most $k$.

\section{REFERENCES}

[1] S. CARTER - Y. KAYA, Immersions with a parallel normal field. Beiträge zur Algebra und Geometrie. Contributions to Algebra and Geometry, 41 (2) (2000), pp. 359-370.

[2] S. CARTER - Y. KAYA, The push-out space of spherically immersed surfaces. Algebras, Groups and Geometries, 18 (4) (2001), pp. 421-433. 
[3] S. CARTER - Z. SENTÜRK, The space of immersions parallel to a given immersion. J. London Math. Soc., 50 (2) (1994), pp. 404-416.

[4] S. Carter - A. West, Partial tubes about immersed manifolds. Geom. Dedicata, 54 (2) (1995), pp. 145-169.

[5] I. Madsen - J. Tornehave, From Calculus to Cohomology. De Rham cohomology and characteristic classes. Cambridge University Press, 1997.

[6] J. Milnor, Morse Theory. 5th ed., Princeton University Press, New Jersey, 1973.

[7] K. Nomizu, Characteristic roots and vectors of a differentiable family of symmetric matrices. Lin. and Multilin. Alg., 2 (1973), pp. 159-162.

[8] R. S. Palais - C. L. Terng, Critical Point Theory and Submanifold Geometry. Lecture Notes in Mathematics, 1353, Springer-Verlag, Berlin, 1988.

Manoscritto pervenuto in redazione il 24 maggio 2010. 